\title{
Supporting information \\ Noninvasive in vivo imaging and monitoring of 3D-printed polycaprolactone scaffolds labeled with an NIR region II fluorescent dye
}

Linzhi Jing a,c, Mingtai Sun a , Pingkang Xu ${ }^{\text {a,c }}$, Kai Yao ${ }^{b}$, Jiao Yang d Xiang Wang c, Hang Liu ${ }^{\mathrm{a}, \mathrm{c}}$, Minxuan Sun ${ }^{\mathrm{d}}$, Yao Sun ${ }^{\mathrm{e}}$, Runyan $\mathrm{Ni}^{*}$,a , Jie Sun ${ }^{*}$,b and Dejian Huang ${ }^{*}, \mathrm{a}, \mathrm{c}$

a) National University of Singapore (Suzhou) Research Institute, 377 Linquan Street, Suzhou, Jiangsu 215123, China

b) Department of Mechatronics and Robotics, Xi'an Jiaotong-Liverpool University, 111 Ren'ai Road, Suzhou, Jiangsu 215123, China

c) Department of Food Science and Technology, National University of Singapore, Science Drive 2, Singapore 117542, Singapore

d) Key Laboratory of Medical Optics, Suzhou Institute of Biomedical Engineering and Technology, Chinese Academy of Science, 88 Keling Road, Suzhou, Jiangsu 215123, China

e) Key Laboratory of Pesticides and Chemical Biology, Ministry of Education, College of Chemistry, Central China Normal University, 152, Luoyu Road, Wuhan, Hubei, 430079, China

* To whom correspondence should be made. Runyan Ni, email: runyan.ni@nusri.cn. Jie Sun, email: Jie.Sun@xjtlu.edu.cn.

Dejian Huang, email: fsthdj@nus.edu.sg.

\section{Keywords}

NIR-II, noninvasive, electrohydrodynamic jet printing, scaffold implants, polycaprolactone 


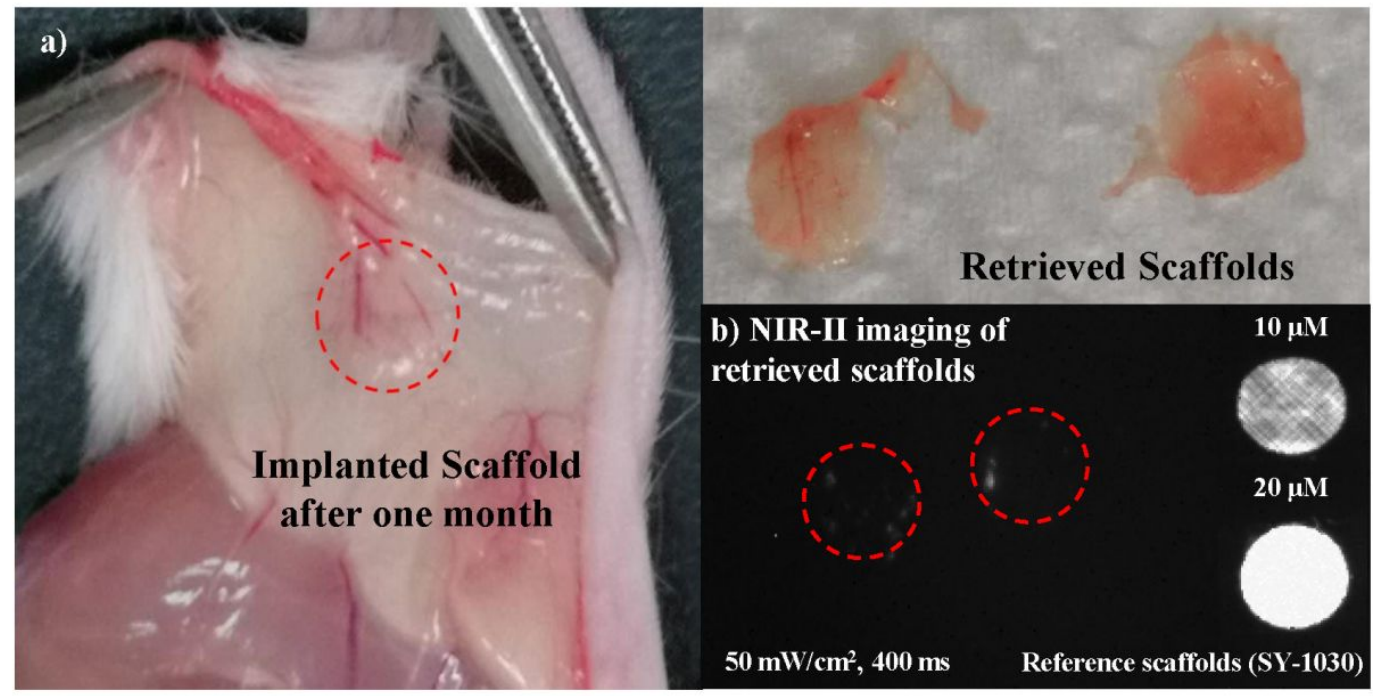

Figure S1. a) Optical images of PCL scaffolds (containing SY-1030 dye) embedded in the skin tissue and stripped scaffolds with the ingrowth of connective tissues and blood vessels. After 1 month implantation. The red circles point out the position of scaffolds. b) NIR-II imaging of retrieved PCL scaffolds at laser current of $50 \mathrm{~mW} / \mathrm{cm}^{2}$ and exposure time of $400 \mathrm{~ms}$. 


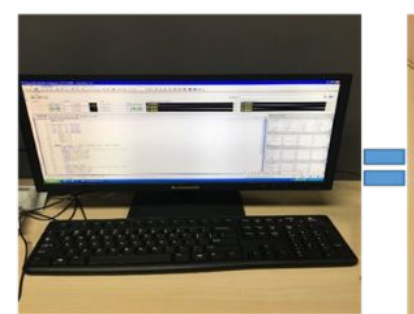

Programming Software

High Voltage source 0-10 kV
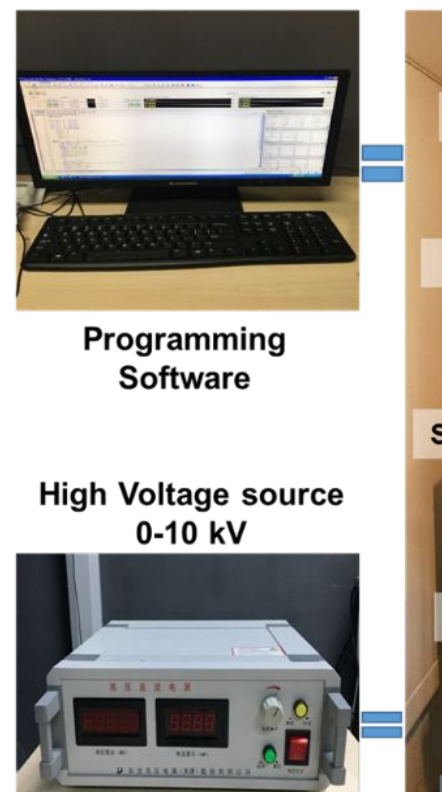

Enclosure
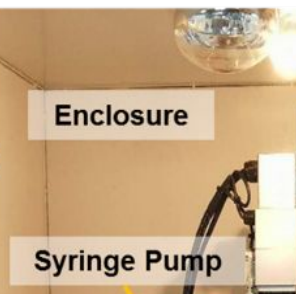

Infrared lamps

(1)

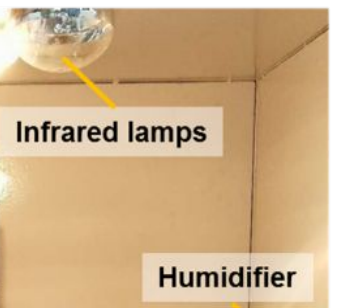

Humidifier

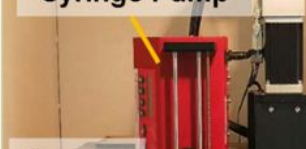

Syringe
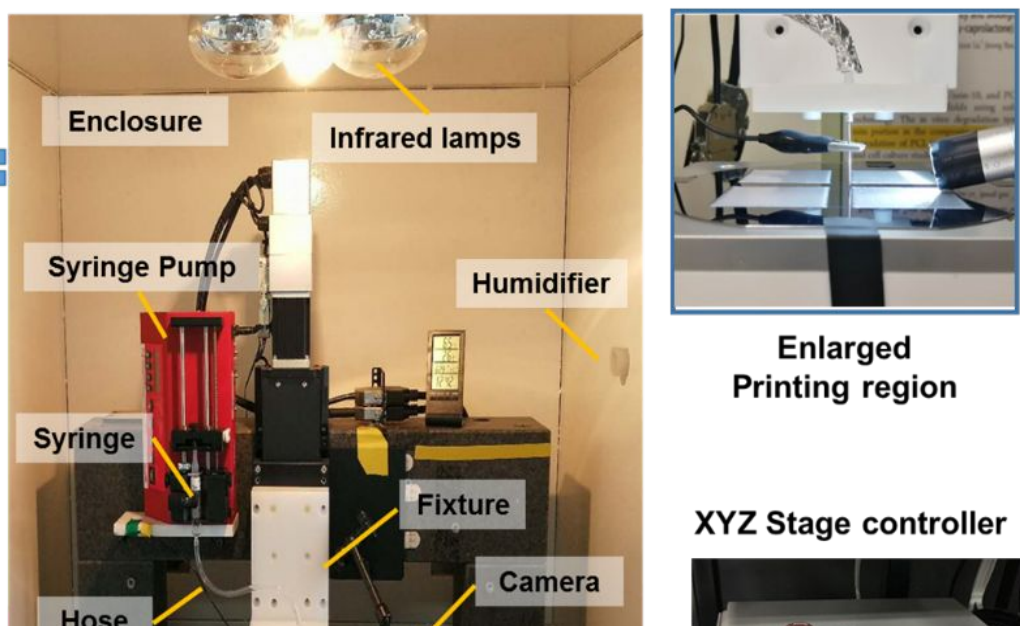

Enlarged

Printing region

XYZ Stage controller

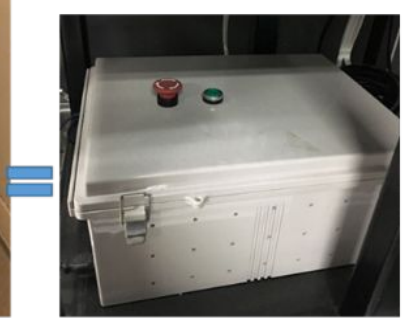

Figure S2. A homemade solution-based EHDJ printing system with temperature, humidity control and printing monitoring system.

Table S1. Printing parameters for EHDJ printing of pure PCL ink and PCL inks containing SY-1030 and SY-COO-PCL

\begin{tabular}{|c|c|c|c|}
\hline & PCL & $\begin{array}{c}\text { PCL (SY-1030, } 20 \\
\mu \mathrm{M})\end{array}$ & $\begin{array}{c}\text { PCL (SY-COO-PCL, } \\
8 \mu \mathrm{M})\end{array}$ \\
\hline \multicolumn{4}{|l|}{ Solution feeding rate } \\
\hline$(\mu \mathrm{L} / \mathbf{m i n})$ & 1.50 & 1.30 & 1.10 \\
\hline $\begin{array}{c}\text { Applied voltage } \\
(\mathrm{kV})\end{array}$ & 2.00 & 1.95 & 1.90 \\
\hline $\begin{array}{l}\text { Nozzle-to-substrate distance } \\
\qquad(\mathbf{m m})\end{array}$ & 2.0 & 2.0 & 2.0 \\
\hline $\begin{array}{l}\text { Stage speed } \\
(\mathrm{mm} / \mathrm{s})\end{array}$ & 150 & 150 & 150 \\
\hline $\begin{array}{l}\text { Temperature } \\
\quad\left({ }^{\circ} \mathrm{C}\right)\end{array}$ & $24.3 \pm 2.0$ & $24.6 \pm 2.0$ & $24.2 \pm 2.0$ \\
\hline $\begin{array}{c}\text { Relative Humidity } \\
\text { (\%) }\end{array}$ & $55 \pm 5$ & $55 \pm 5$ & $55 \pm 5$ \\
\hline
\end{tabular}


Table S2. Morphological data of PCL scaffolds with NIR-II dyes

\begin{tabular}{ccc} 
Parameters & PCL $($ SY-1030) & PCL (SY-COO-PCL) \\
Pore size $(\boldsymbol{\mu m})$ & $170.8 \pm 9.2$ & $172.3 \pm 11.5$ \\
Top fiber width $(\boldsymbol{\mu m})$ & $14.7 \pm 1.1$ & $15.1 \pm 0.8$ \\
Bottom fiber width $(\boldsymbol{\mu m})$ & $30.5 \pm 6.1$ & $27.3 \pm 5.9$ \\
Overall thickness $(\boldsymbol{\mu m})$ & $177.6 \pm 22.5$ & $187.4 \pm 24.9$ \\
& & $87.9 \pm 0.5$ \\
\hline
\end{tabular}




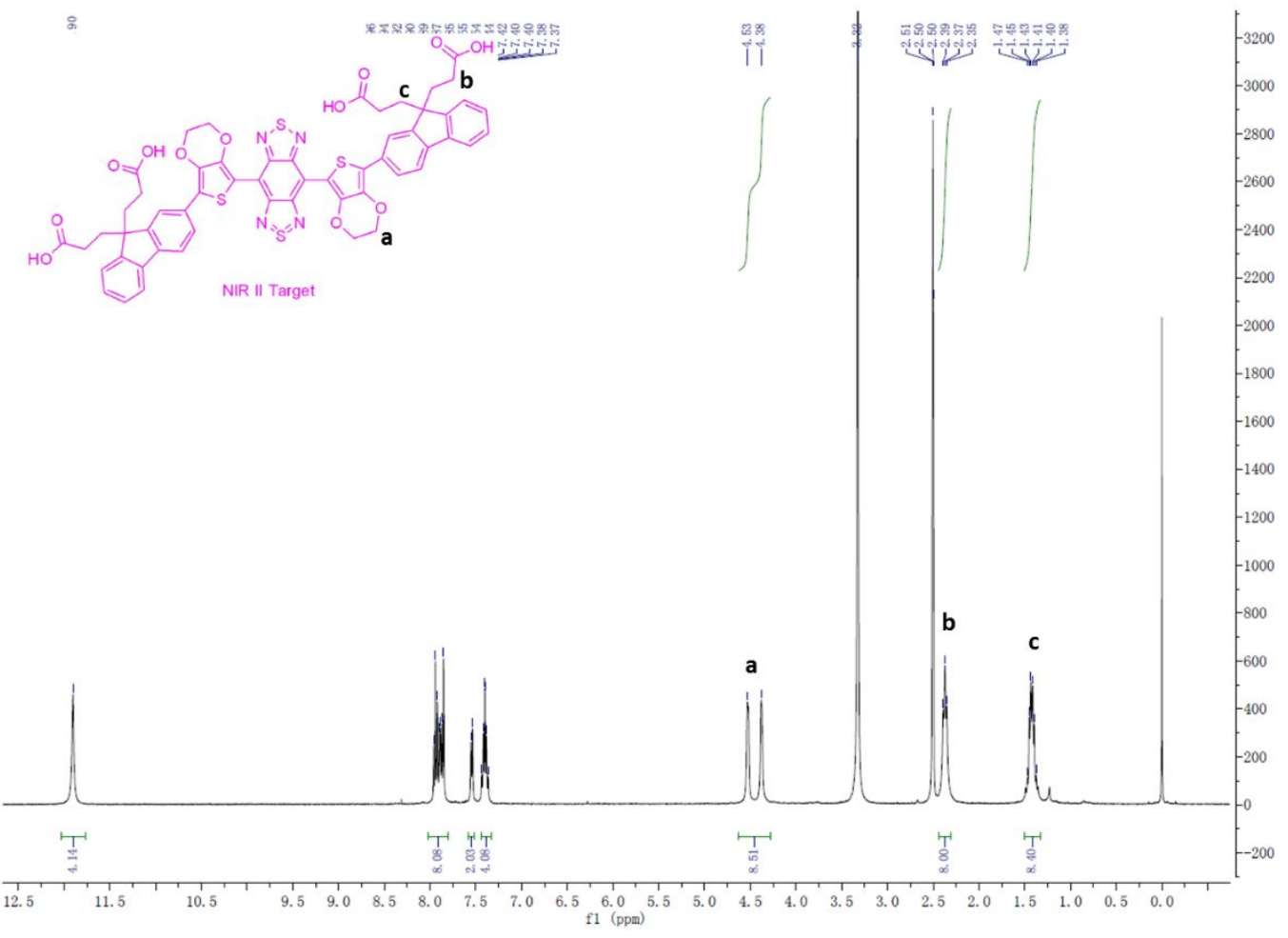

Figure. S3 ${ }^{1} \mathrm{H}-\mathrm{NMR}$ spectra of SY-COOH (500 MHz, DMSO-d ${ }_{6}$ ).

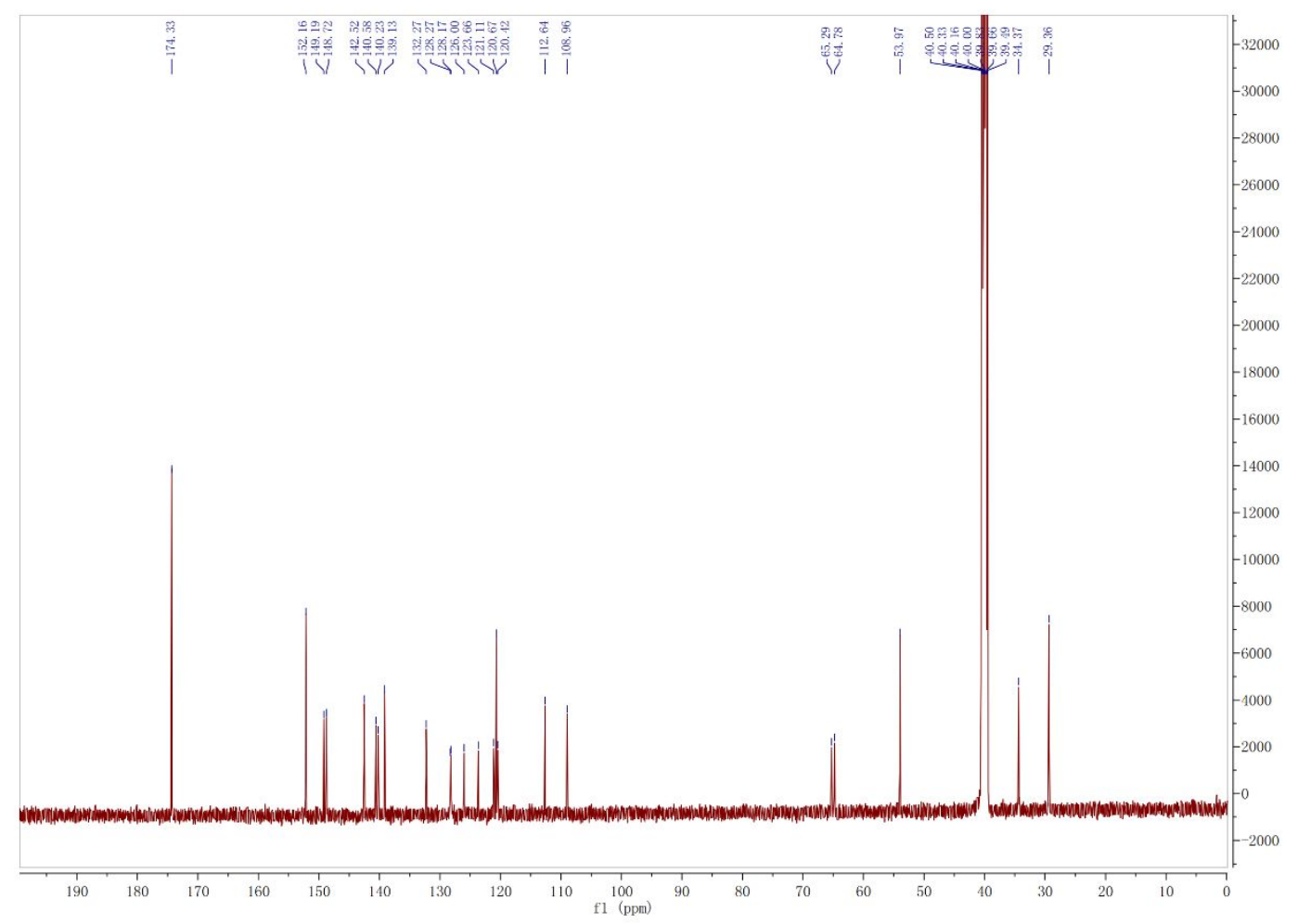

Figure S4. ${ }^{13} \mathrm{C}-\mathrm{NMR}$ spectra of SY-COOH (125 MHz, DMSO-d $\left.{ }_{6}\right)$. 


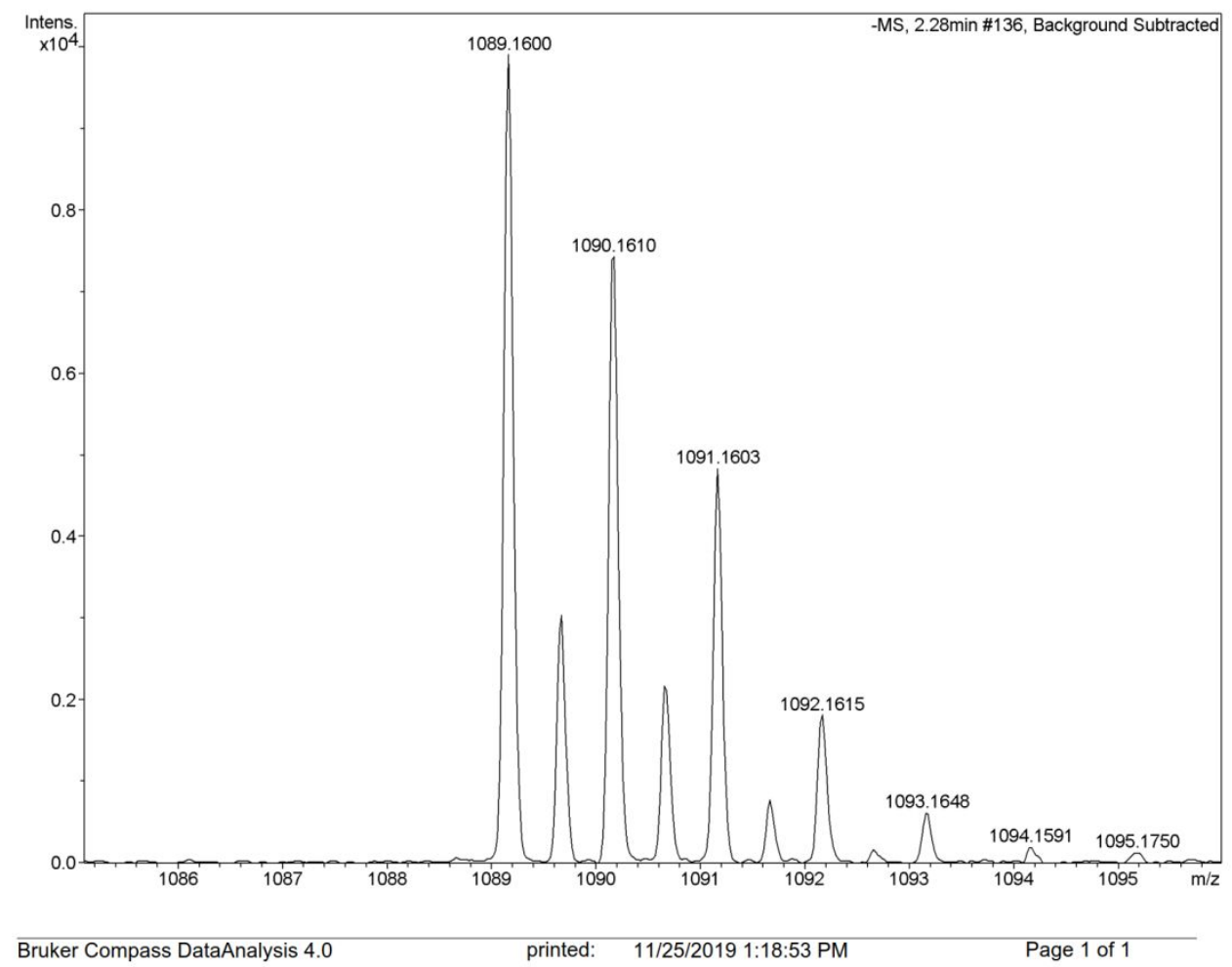

Figure S5. High-resolution mass spectra of SY-COOH (Electrospray ionization).

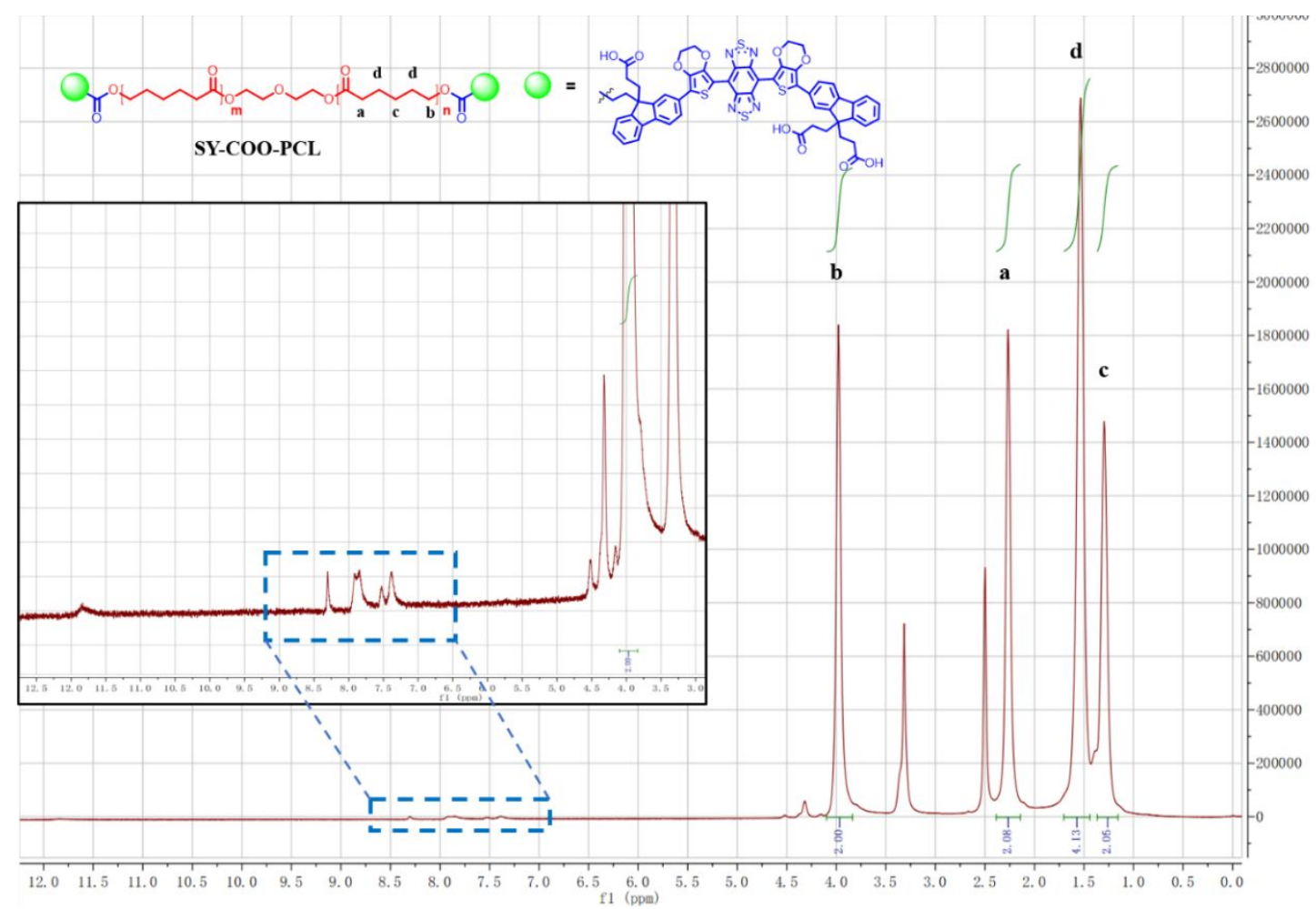

Figure. S6 ${ }^{1} \mathrm{H}-\mathrm{NMR}$ spectra of SY-COO-PCL (500 MHz, DMSO-d 6 ). 